$43(88 \%)$ of the students wanted these topics covered during medical school. A recent survey of 56 UK paediatricians and general practitioners rated the importance of undergraduate knowledge of normal feeding $3.9 / 5$ and ability to engage in health promotion $3.8 / 5$.

Conclusions This study demonstrates a lack of confidence and competence among medical students to provide advice about childhood nutrition, despite clinicians considering the topic essential undergraduate knowledge. Concerted efforts must be made to improve the quality of nutrition and health promotion advice to parents and undergraduate training provides an ideal opportunity to develop these skills.

\section{G363(P) INFECTIOUS COMPLICATIONS IN BILIARY ATRESIA; A SINGLE CENTRE EXPERIENCE}

${ }^{1} \mathrm{~V}$ Jain, ${ }^{1} \mathrm{O}$ Kaltsogianni, 'S Bansal, ${ }^{1} \mathrm{M}$ Davenport, ${ }^{1} \mathrm{~A}$ Dhawan, ${ }^{2} \mathrm{~A}$ Verma. ${ }^{1}$ Paediatric Hepatology, Gastroenterology and Nutrition Centre, Kings College Hospital, London, UK; ${ }^{2}$ Institute of Liver Disease, Kings College Hospital, London, UK

\subsection{6/archdischild-2015-308599.319}

Objectives To evaluate incidence and timing of cholangitis and spontaneous bacterial peritonitis (SBP) in Biliary Atresia (BA), after Kasai Porto-enterostomy (KP), prior to liver transplantation (LT).

Methods A single-centre retrospective analysis, comprising 78 patients (36M:42F) who underwent KP between 2008-2010.

Results Cholangitis followed in 38/78 (48\%) patients; median number of episodes 2 (range: 1,5 ). Median age for first episode was 5.6 months $(2,72.5)$. Six patients showed dilated biliary radicles on ultrasound. Organisms were isolated from blood cultures in six patients; E-coli $(\mathrm{n}=2)$, Staphylococcus Aureus, Klebsiella, Streptococcus Pneumonia and Pseudomonas. 27/38 (71\%) cholangitic patients underwent LT, 10 are alive with their native liver and one died. Ascites developed in 29/78 (37\%) patients, at median age 6.5 months $(3.1,66)$. Ascitic taps were performed in $41 \%(12 / 29)$, due to respiratory distress with fever $(5 / 12)$ or without fever $(7 / 12)$ at median age 7.4 months $(3.2$, 22.8). 16/17 patients that did not have ascitic taps underwent LT at a median age 17.2 months $(7.4,79.7)$ and one died whilst listed for LT. Timing of tap was at the onset of ascites in six patients and at a median time of 2 months $(0.1,4.6)$ from onset of ascites in the remaining six patients. Four patients fulfilled criteria for SBP diagnosis; 3 culture-negative (wcc $>250 \mathrm{~mm}^{3}$ ), one bacteri-ascites (wcc $<250 \mathrm{~mm}^{3}$; gram-positive cocci, gram-negative rods). No culture-positive SBP was identified. One culturenegative SBP revealed Streptococcus Pneumoniae in blood cultures. Five patients that underwent ascitic taps previously had cholangitis. Antibiotics were already commenced in $8 / 12$ patients pre-tap. Raised plasma wcc $\left(>17 \mathrm{~mm}^{3}\right)$ was identified in SBP (3/4) and non-SBP (3/8) patients. All SBP patients underwent LT at a median age 10.5 months $(7.1,16.1)$. Non-SBP patients underwent LT $(\mathrm{n}=4)$, are alive with native liver $(\mathrm{n}=1)$ or died $(\mathrm{n}=3$; all listed for LT).

Conclusion Cholangitis and SBP occurred in $48 \%$ and $5 \%$ of BA patients respectively; cholangitis episodes presented earlier. Few cases revealed positive bacterial cultures. Definition of paediatric SBP needs to account for pre-culture antibiotic use. New molecular techniques should be sought to aid diagnosis. LT is a successful outcome for cholangitis, SBP and non-SBP ascites.

\section{G364(P) MANAGEMENT OF GASTROESOPHAGEAL REFLUX IN INFANTS: CURRENT PRACTICE OF DIAGNOSIS AND TREATMENT IN A UK DISTRICT GENERAL HOSPITAL}

ST Belitsi, A Varghese Mathew, M James. Paediatrics and Neonates, Ipswich Hospital NHS Trust, Ipswich, UK

\subsection{6/archdischild-2015-308599.320}

Objectives and study According to current ESPGHAN and NASPGHAN guidelines, gastroesophageal reflux (GER) is defined as the passage of gastric contents into the oesophagus with or without regurgitation and vomiting. GER is considered to be physiological in the majority of young infants under the age of 12 months. Typically, episodes of GER in healthy individuals last $<3 \mathrm{~min}$, occur in the postprandial period, and cause few or no symptoms. In contrast, gastroesophageal reflux disease (GERD) is present when the reflux of gastric contents causes troublesome symptoms and/or complications such as faltering growth, recurrent pneumonias, apnoea, dystonic head posturing. Limited data is currently available on the adherence of healthcare professionals to existing guidelines in daily practice, however some reports highlight the risk of over-diagnosing GERD and the inadequate use of treatments, such as acid suppression. Aim of the study The aim of this study was to assess current clinical practice and adherence to ESPGHAN guidelines regarding the diagnosis and management of infants presenting with symptoms of GER/GERD.

Methods Medical records of 60 patients treated in an inpatient or outpatient setting, age 1 day to 1 year and with a diagnosis of GOR or GORD were randomly collected. Data recorded for each patient included: specific diagnosis, presence of clinical symptoms suggestive of GOR/GORD, choice of treatment and if the diagnostic criteria according to ESPGHAN guidelines were met.

Results In total $100 \%$ of patients included in this study were diagnosed with GOR, rather than GORD despite the presence of what could be considered as "troublesome symptoms". The two most frequently reported symptoms were poor weight gain $\mathrm{n}=6(10 \%)$ and irritability $\mathrm{n}=40(66 \%)$. Importantly, despite being "officially" diagnosed with GOR, 55 out of 60 children (91\%) were commenced on anti-reflux medication (i.e. proton pump inhibitors or the $\mathrm{H} 2$ receptor blocker Ranitidine). All parents were provided with education and guidance.

Conclusion Our small study provides insight into current clinical practice in a district general hospital and highlights major insufficiency in the understanding and management of GOR/GORD. Despite diagnosing GOR the majority of infants are commenced on medical treatment. Active advice and teaching to healthcare professionals in the primary and secondary sector is required to improve the situation in the long term.

\section{G365(P) THE USE OF ANTIBIOTIC LINE LOCKS TO PREVENT CENTRAL VENOUS CATHETER-ASSOCIATED SEPSIS IN CHILDREN WITH INTESTINAL FAILURE ON LONG-TERM PARENTERAL NUTRITION}

\begin{abstract}
${ }^{1,2} \mathrm{~B}$ Morrissey, ${ }^{3} \mathrm{E}$ Hennessy, ${ }^{2} \mathrm{~S}$ Naik, ${ }^{2} \mathrm{~N}$ Meadows, ${ }^{4} \mathrm{M}$ Millar. ${ }^{1}$ Paediatrics, Homerton University Hospital NHS Trust, London, UK; ${ }^{2}$ Paediatric Gastroenterology, Barts Health NHS Trust, London, UK; ${ }^{3}$ Wolfson Institute, Barts and The London School of Medicine and Dentistry, Queen Mary University of London, UK; ${ }^{4}$ Microbiology, Barts and The London School of Medicine and Dentistry, Queen Mary University of London, UK
\end{abstract}

10.1136/archdischild-2015-308599.321 